\title{
Drug Binding Site
}

National Cancer Institute

\section{Source}

National Cancer Institute. Drug Binding Site. NCI Thesaurus. Code C13405.

The reactive parts of a macromolecule that directly participate in its specific combination with a drug. 